\title{
Electrical Drive Radiated Emissions Estimation in Terms of Input Control Using Extreme Learning Machines
}

\author{
A. Wefky, F. Espinosa, L. de Santiago, P. Revenga, \\ J. L. Lázaro, and M. Martínez \\ Electronics Department, Polytechnics School, University of Alcalá, Campus Universitario 28871, \\ Alcalá de Henares, Spain \\ Correspondence should be addressed to F. Espinosa, espinosa@depeca.uah.es
}

Received 21 September 2012; Revised 9 November 2012; Accepted 9 November 2012

Academic Editor: Wuhong Wang

Copyright (C) 2012 A. Wefky et al. This is an open access article distributed under the Creative Commons Attribution License, which permits unrestricted use, distribution, and reproduction in any medium, provided the original work is properly cited.

With the increase of electrical/electronic equipment integration complexity, the electromagnetic compatibility (EMC) becomes one of the key points to be respected in order to meet the constructor standard conformity. Electrical drives are known sources of electromagnetic interferences due to the motor as well as the related power electronics. They are the principal radiated emissions source in automotive applications. This paper shows that there is a direct relationship between the input control voltage and the corresponding level of radiated emissions. It also introduces a novel model using artificial intelligence techniques for estimating the radiated emissions of a DCmotor-based electrical drive in terms of its input voltage. Details of the training and testing of the developed extreme learning machine (ELM) are described. Good agreement between the electrical drive behavior and the developed model is observed.

\section{Introduction}

Because of the integration density increase and the assembly of electric/electronic circuits gathered in a confined space such as electrical vehicles, certain unwanted effects caused by EMC and electromagnetic interference (EMI) become unavoidable [1-3]. Electrical drives are the main source of electromagnetic emissions of electrical vehicles, including both electrical motor and the required power electronics. Indeed, the addition of equipment operating in fast switching is susceptible to cause harmful conducted and/or radiated interference that can spread through the onboard network [4]. These coupling effects may damage the electrical module in which they were integrated as can be found in digital and mixed-signal electronic circuits such as the RF devices and microcontrollers integrated in the command devices [5]. 
To predict the various EMI effects generated by electrical motors, different models of machine windings [6] and magnets [7] using lumped elements [8] were proposed. The influences of an innovative mechanism of collector-brush contact on the EMC model of DC motors have been investigated in [9].

ELMs are one of the most powerful emergent tools that have been widely used in recent years in various fields [10] such as security assessment [11], data privacy [12], EEG and seizure detection [13], image quality assessment [14], implementation with FPGAs [15], face recognition [16], and human action recognition [17]. Function approximation is one of the basic learning tasks that an ELM can accomplish [18]. This paper exploits this capability to estimate the drive's radiated emissions in terms of its input voltage.

The Electronics Department at the University of Alcala (UAH), in collaboration with the Thermal Engines Group of the ETSII-UPM in Madrid and the Research Center for Environmental Energy and Technology (CIEMAT) in Madrid developed an electronic measurement equipment to relate the drive activity, vehicle state, and road conditions with pollutant emissions (gases and particles) in real traffic conditions [19]. This was the starting point to extend the study to radiated electromagnetic emissions. In order to start the modeling process with a basic configuration, this work concentrates on the effect of the input voltage of an electrical drive based on a DC motor on the drive radiated emissions.

This paper is arranged as follows. Section 2 highlights the context of this work by discussing the relationship between the driving profile and the vehicular radiated emissions. Section 3 describes the key aspects of the measurement methodology followed in the experimental tests. Details of ELM model development are discussed in the Section 4. Section 5 is devoted to commenting on the obtained experimental results. Finally, conclusions and future work are included in Section 6.

\section{Driving Profile versus Vehicular Radiated Emissions}

Current EMC standards, like CISPR 16-2-3 [20], do not discuss the effect of the driving characteristics on real traffic vehicular radiated emissions. There are a lot of driving signals that can describe the driving style such as vehicle speed, linear acceleration, frontal inclination, regime engine, following distance, relative lane position, yaw angle, position of throttle, clutch, and brake pedals [21, 22].

A straightforward solution to study the effect of the driving profile on the radiated emissions could be to measure a set of driving profile signals as well as EMI signals simultaneously in real traffic conditions. Measuring some driving profile signals in real time is an easy task because in most cases the onboard electronic system can easily provide information about most of these variables. On the other hand, measuring radiated electromagnetic emissions due to a specific electric vehicle in real traffic seems to be a very complicated task. This is because the onboard antenna that would receive the radiated emissions due to the vehicle under test (VUT) only would receive EMI signals from many other sources like radiated emissions coming from other vehicles, WiFi, AM radio, FM radio, television broadcast, mobile networks, satellite networks, Bluetooth devices, GPS, high voltage towers, and so forth. Mainly for this reason, but also for the size and weight of the required antennas for this purpose, it is impossible to measure the real traffic vehicular radiated emissions due to a specific VUT. Thus, the development of a model that would be able to estimate real traffic vehicular radiated emissions in terms of the corresponding driving style signals would be a novel work. In this way, the relative change of the real traffic radiated 
emissions in terms of the driving profile parameters can be quantified. Therefore, guidelines can be determined in order to ensure green driving profiles in terms of minimization of vehicular radiated emissions.

In order to estimate the vehicular real traffic radiated emissions in terms of the driving behaviour signals, authors propose a process of three main stages as shown in Figure 1. Firstly, tests with the vehicle in a semianechoic chamber have to be done measuring some driving profile signals as well as the corresponding radiated emissions. Secondly, ELMs should be exploited to develop the desired model using data registered from the previous stage. Thirdly, real traffic experiments have to be done registering only the driving profile signals that will be simultaneously applied to the obtained model estimating the real traffic radiated emissions in terms of the registered real traffic driving profile signals.

As the electrical drive of electric vehicles is one of the most powerful sources of radiated emissions [23], the authors in this work have applied the first two steps of the above algorithm on an electrical drive as a first approach, that is, a DC-motor-based electrical drive has been tested in a semianechoic chamber measuring its input voltage that emulates the driving profile signals as well as its corresponding radiated emissions. Then, the authors have developed a neural network model that is capable of estimating the radiated emissions of the electrical drive in terms of the corresponding input voltage.

As a first trial, the authors presented a measurement methodology of the radiated emissions of electric vehicles as well as the driving profile [24]. This methodology is based on frequency domain EMI measurement procedure where a spectrum analyzer has been used to make sweeps of the radiated emissions signal. In the present work, the authors propose a time domain EMI measurement system based on a digital oscilloscope in order to save the overall measurement time.

\section{Measurement Methodology}

The basic idea of the proposed methodology is shown in Figure 2. Firstly, the EMI signal of the drive under test (DUT) is captured by a biconical antenna, filtered by an antialiasing lowpass filter, sampled as well as quantized by the digital oscilloscope's ADC, and sent to the converter via the GPIB bus which delivers it to the PC's USB port to be saved in a database for offline processing. The previous scenario is repeated until all the time domain sweeps are saved in the PC's database.

At the end of the experiment, spectrum and spectrogram of the electrical drive's EMI signal are computed via the discrete Fourier transform (DFT). Moreover, the time domain evolution of the electrical drive's radiated emissions is displayed with the input voltage profile applied to the electrical drive.

According to the measurement scenario described in the previous section, the digital oscilloscope provides a matrix of radiated emission sweeps. Then, the sweep mean power (SMP) is calculated as follows:

$$
\mathrm{SMP}=\frac{\left(\left(1 / N_{s}\right) \sum_{i=1}^{N_{s}} v_{i}^{2}\right)}{50},
$$

where $N_{s}$ is the sweep length, $i$ is the sweep sample index, and $v$ is the sweep sample value in Volts. 


\section{(1) Realization of real experiments in a semianechoic chamber}

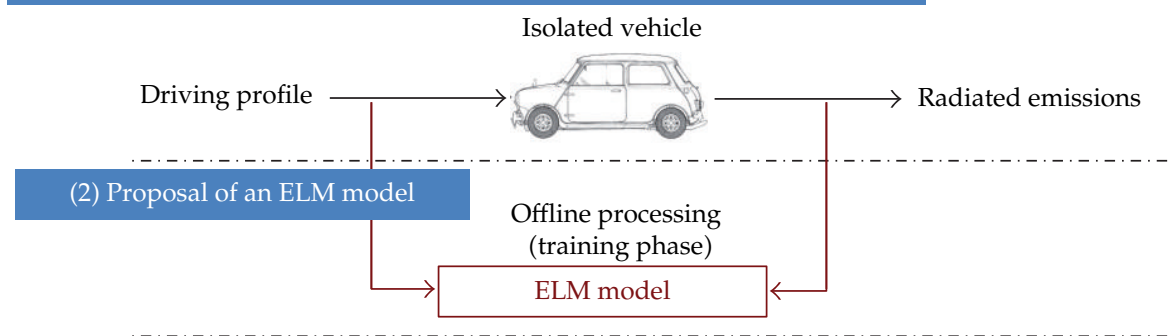

(3) Experimental validation

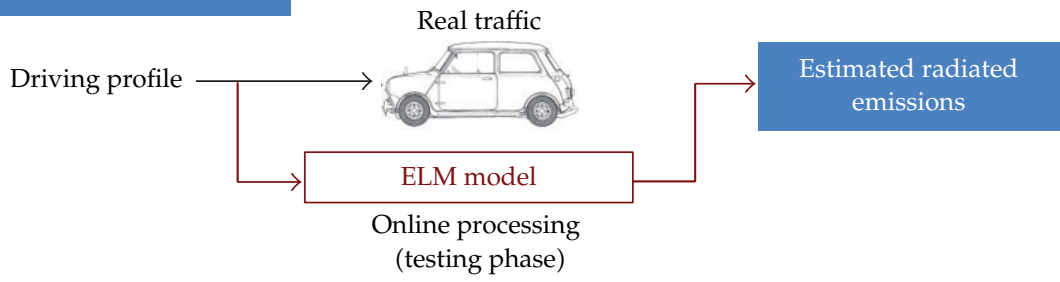

Figure 1: Complete process for estimating real traffic vehicular radiated emissions in terms of the driving profile.

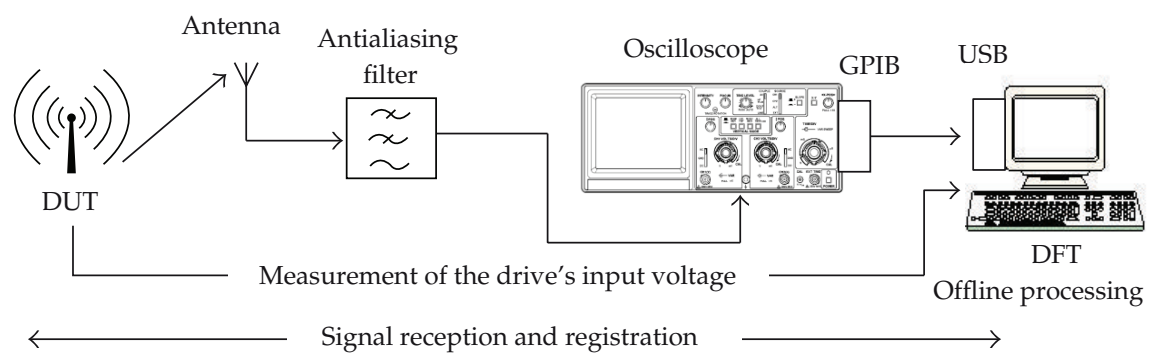

Figure 2: Block diagram of the proposed TDEMI measurement system.

The frequency range of interest in the study must be covered by the antenna bandwidth. In order to meet the sampling theorem and to avoid the aliasing effect, an antialiasing low-pass filters (LPF) must be connected to the antenna.

The following parameters of the oscilloscope should be properly adjusted: sampling time $T_{s}$ (or the sampling frequency $F_{s}$ ), capture time $T_{c}$ (duration of the time domain sweep of the oscilloscope), and sweeping time $T_{\mathrm{sw}}$ (or the sweeping frequency). The relation between the sampling, sweeping, and capturing times is illustrated in Figure 3.

According to the sampling theorem, the minimum sampling frequency equals twice the wanted maximum frequency (Nyquist frequency) of the calculated spectrum. To avoid aliasing errors, it is recommended to set the sampling frequency 2 to 4 times higher than Nyquist frequency depending on the steepness of the antialiasing filter. The sampling frequency used in this work was $12.5 \mathrm{MHz}$ with an antialiasing filter of $1.9 \mathrm{MHz}$ cutoff frequency. 


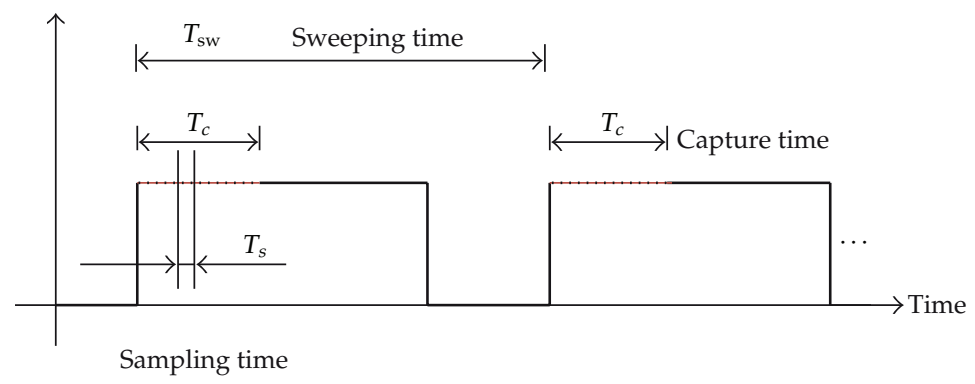

Figure 3: The relation between the sampling, sweeping, and capture times.

Besides, the capture time $T_{c}$ depends on the distance between two neighboring frequency bins (frequency resolution or frequency step) $\Delta f$ which depends on the resolution bandwidth $B_{r}$ as follows [25-28]:

$$
T_{c}=\frac{1}{\Delta f}=\frac{1}{1.06 B_{r}}
$$

As a result of choosing these two parameters, that is, $F_{s}$ and $T_{c}$, the time domain sweep length $N_{S}$, or number of samples per sweep is determined as follows:

$$
N_{s}=F_{S} \times T_{c}
$$

It is noteworthy that the sweep length is limited by the oscilloscope's available memory as well as the maximum data transmission speed between the oscilloscope and the personal computer. As can be seen from (3.3), the sweep length depends on both the sampling frequency and the capture time. The capture time is already set by the resolution bandwidth determined by the currently available standards as can be seen in (3.2). On the other hand, the sampling frequency can be more than twice the maximum measured signal bandwidth. Thus, the lower bound on the sampling frequency is already imposed by the sampling theorem. Consequently, the upper bound on the sampling frequency is the value at which the oscilloscope's memory saturates or the maximum transmission speed between the oscilloscope and the PC is reached.

Finally, the sweeping time $T_{\mathrm{sw}}$ (time between the start of two consecutive records), or the recording frequency $F_{\mathrm{sw}}$, has to be adjusted based on the profile of the drive input voltage and the maximum transmission speed between the PC and the oscilloscope. In other words, the details and changes included in the input voltage profile of the DUT impose an upper bound on the recording time, that is, the maximum separation between the starting of consecutive records has to be less than the minimum separation between two successive important changes in the input voltage profile of the DUT.

Moreover, the maximum data transmission speed between the oscilloscope and the PC passing by the GPIB/USB converter controls the lower limit on the recording time $T_{r}$. In other words, the minimum recording time corresponds to the maximum data transmissions speed between the oscilloscope and the PC.

The proposed TDEMI system has been validated against an ESIB26 EMI receiver from Rhode and Schwarz, comparing the spectra obtained by both systems due to emissions of the 
Table 1: Comparison between generalization RMSE corresponding to different hidden neurons.

\begin{tabular}{lccccccc}
\hline Hidden neurons & 2 & 4 & 10 & 15 & 20 & 25 & 30 \\
Testing RMSE $* 1 e-8$ & 1.3127 & $\mathbf{1 . 2 8 2 2}$ & 1.3363 & 1.3751 & 1.4408 & 1.3689 & 1.6281 \\
\hline
\end{tabular}

same square wave signal source. The validation results showed the agreement between the spectra obtained by the TDEMI system and the EMI receiver. It is noteworthy that the authors in this work are not interested in obtaining accurate absolute levels of radiated emissions. On the other hand, they are interested in measuring the relative change in the level of radiated emissions due to the change in the applied voltage.

\section{Extreme Learning Machines}

Artificial intelligence techniques have been used in various applications. Among different computational intelligence techniques, ELM is considered a recently emerging technique that overcomes some challenges faced by artificial neural networks (ANNs) and support vector machines (SVMs) such as slow learning speed, trivial human intervene, and poor computational scalability [29]. Moreover, ELMs satisfy the universal approximation property [30].

When a particular ELM model fails, it could be due to one of two reasons. The model parameters fail to converge to the proper values, perhaps due to unsuitable model initialization, or the inability of the given model to implement the desired function, perhaps due to an insufficient number of hidden neurons. In this work, to avoid the first possibility, each ELM model was trained and tested 50 times. And the network architecture with the lowest root mean square error (RMSE) on the testing data set has been chosen.

Concerning the second reason, there is no theory yet to explain how many hidden neurons are needed to approximate any given function. If there are too few hidden neurons, a high training error and high generalization error would result from underfitting. On the other hand, if there are too many hidden neurons, there would be a low training error, but there would still be a high generalization error due to overfitting. In most situations, there is no way to determine the best number of hidden neurons without training several networks and estimating the generalization error of each [30-33]. In this paper, the network growing technique [31] is applied by adding hidden neurons sequentially from 1 to 30 comparing the testing RMSE error.

Table 1 shows that 4 hidden neurons have achieved the best generalization performance in terms of the testing RMSE calculated as follows:

$$
\mathrm{RMSE}=\left[\frac{1}{N} \sum_{i=1}^{N}\left(P_{i}-O_{i}\right)^{2}\right]^{1 / 2},
$$

where $O$ is the vector of observed (measured) values, $P$ is the vector of model estimated values, and $N$ is the number of samples in the testing subset. The input to the ELM model is the drive input voltage, while the output is its radiated emissions. Therefore, this paper presents a single-input single-output ELM model whose structure is shown in Figure 4. 


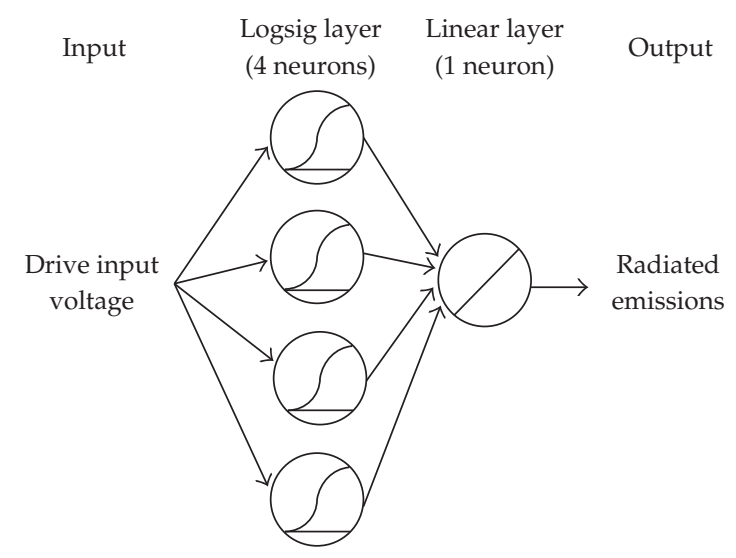

Figure 4: Structure of the ELM network.

\section{Experimental Results}

Experimental tests have been realized in the semianechoic chamber of the High Technology and Homologation Centre at the University of Alcalá. Figure 5 shows a part of the semianechoic chamber with experimental setup used in this work. It shows the biconical antenna applied to receive the electrical drive's radiated emissions as well as the DUT composed of a DC motor and a power electronics card.

The electrical drive has been subjected to two voltage profiles as can be seen in Figures 6 and 7. Radiated emissions registered by the proposed TDEMI system have been processed to calculate both the spectrograms shown in Figures 8 and 9, as well as the SMPs shown in Figures 6 and 7, using (3.1).

The data of the peaks profile, described in Figure 6, has been used in the training phase of the ELM network. This Figure shows that the ELM model has successfully learned to estimate the SMP of the EMI signal in terms of the corresponding input voltage value.

However, the steps profile data, depicted in Figure 7, has been exploited in the testing phase. It also shows that the developed ELM model has been successfully and accurately estimated the SMP of the radiated emissions signal in terms of unseen values of the drive input voltage. The proposed model has been able to precisely distinguish between 8,10 , and 15 volts of the electrical drive excitation.

Figures 8 and 9 illustrate the spectrograms of the radiated emissions EMI signals registered for the peaks and steps driving profiles, respectively. These figures also show a correspondence between the drive input voltage profile and the intensity of the radiated emissions.

\section{Conclusions}

This paper presents a proposal of estimating real traffic vehicular radiated emissions in terms of driving profile signals. The first two steps of this methodology have been applied to an electrical drive based on a DC motor. It has been shown that there is a direct relationship between the electrical drive's input voltage and its corresponding radiated emissions. An 


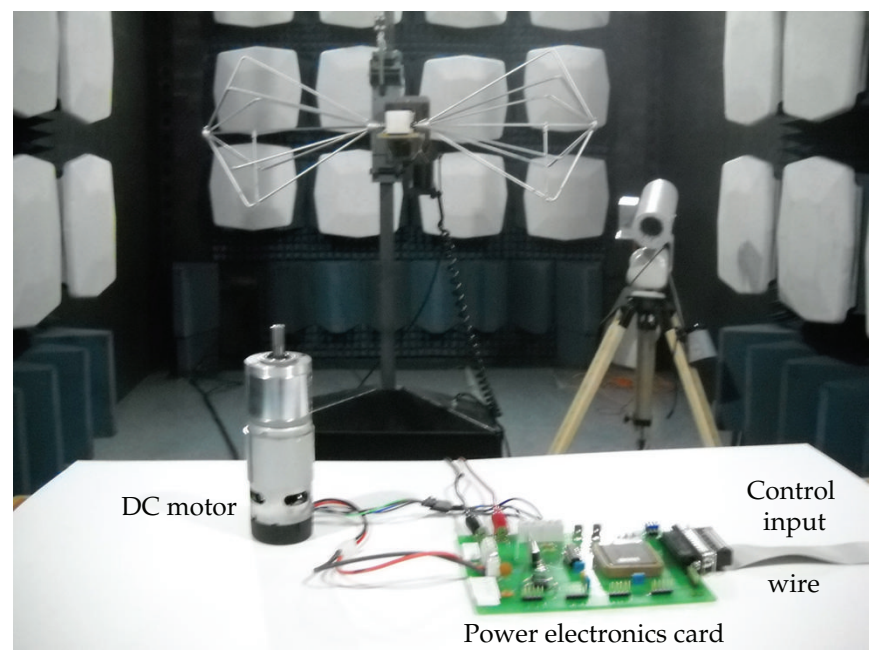

Figure 5: Experimental setup of an electrical drive including a DC motor (left side on the table) and the power electronics card (right side on the table), inside a semianechoic chamber.

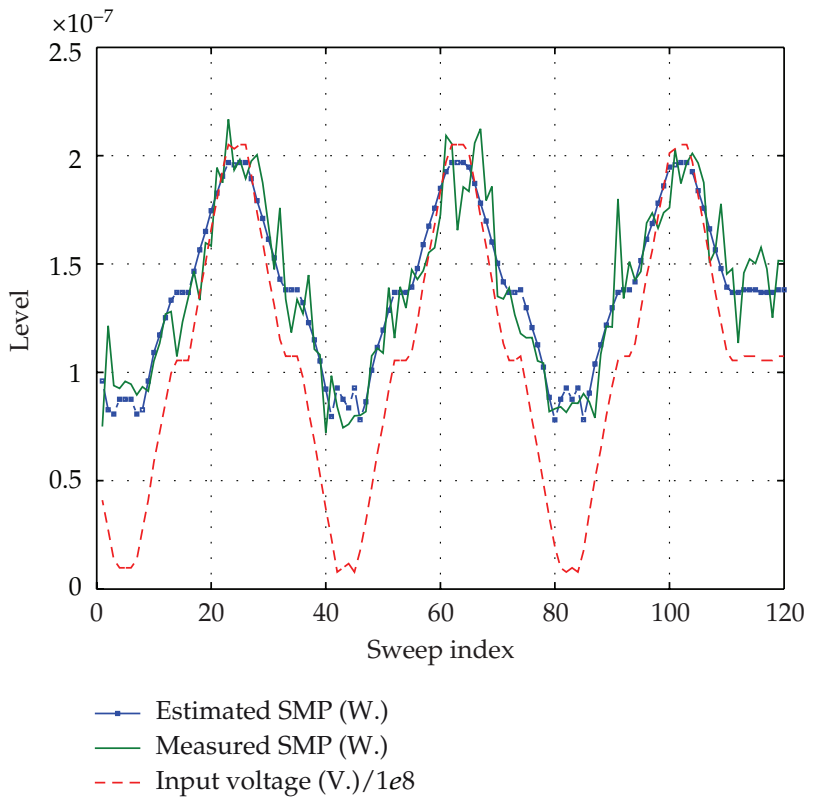

Figure 6: Training data (peaks profile).

ELM model with 4 hidden neurons has been developed to estimate the radiated emissions in terms of the drive input. The model has been validated with experimental data of a real electrical drive tested in a semianechoic chamber. 


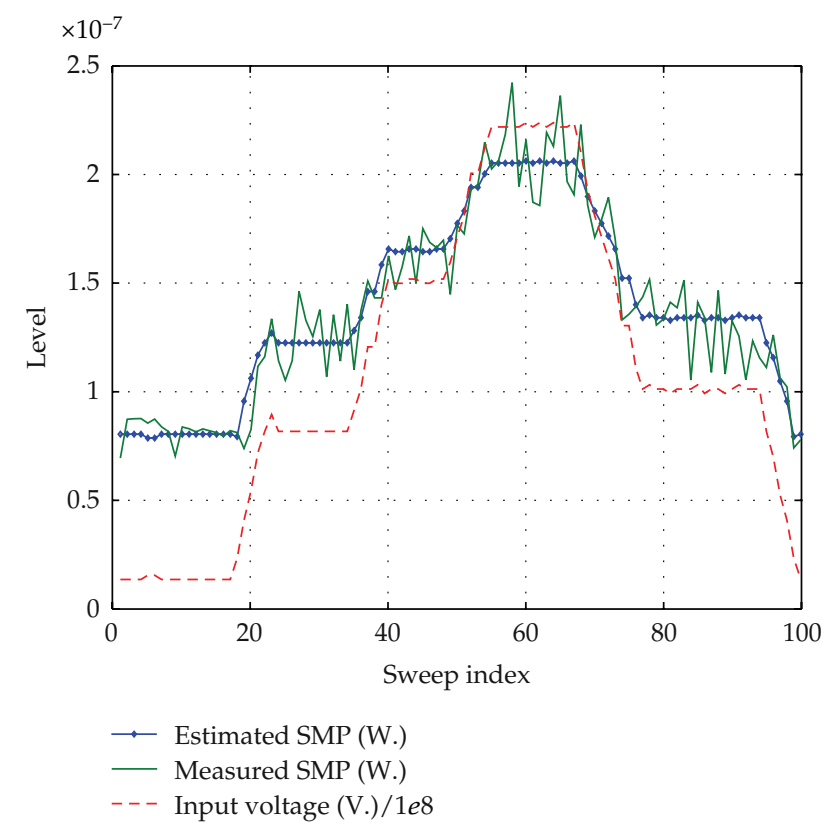

Figure 7: Testing data (steps profile).

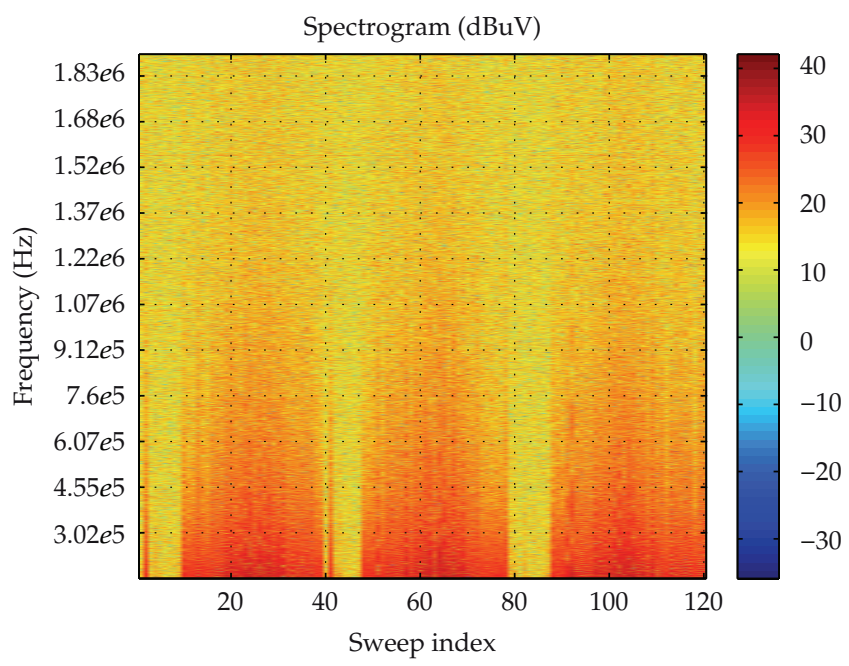

Figure 8: Spectrogram of the radiated emissions EMI signal (peaks profile).

Considering the successfully obtained results, as future work, the authors propose the online application of a model based on ELM to the radiated electromagnetic estimation of electric vehicles, according to the challenge described in Figure 1. 


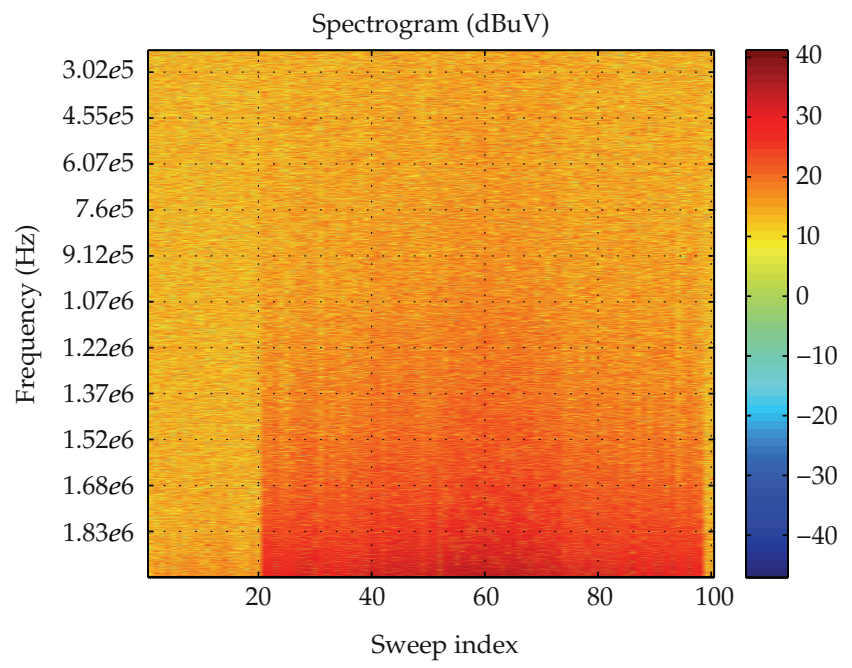

Figure 9: Spectrogram of the radiated emissions EMI signal (steps profile).

\section{Acknowledgment}

The authors would like to acknowledge the cooperation of the High Technology and Homologation Center at the University of Alcalá, Spain.

\section{References}

[1] J. Ben Hadj Slama, S. Hrigua, F. Costa, B. Revol, and C. Gautier, "Relevant parameters of SPICE3 MOSFET model for EMC analysis," in Proceedings of the IEEE International Symposium on Electromagnetic Compatibility (EMC '09), pp. 319-323, August 2009.

[2] S. Chen, T. W. Nehl, J. S. Lai et al., "Towards EMI prediction of a PM motor drive for automotive applications," in Proceedings of the 18th Annual IEEE Applied Power Electronics Conference and Exposition (APEC '03), pp. 14-22, February 2003.

[3] L. Ferrer, J. Balcells, D. González, J. Gago, and M. Lamich, "Modelling of differential mode conducted EMI generated by switched power inverters," in Proceedings of the 29th Annual Conference of the IEEE Industrial Electronics Society (IECON '03), pp. 2312-2315, November 2003.

[4] R. Kahoul, Y. Azzouz, P. Marchal, and B. Mazari, "New behavioral modeling for DC motor armatures applied to automotive EMC characterization," IEEE Transactions on Electromagnetic Compatibility, vol. 52, no. 4, pp. 888-901, 2010.

[5] W. Valente, M. H. Amaral, and A. Raizer, "EMC management: how to compare electromagnetic environmental measurements and equipment immunity levels," Progress In Electromagnetics Research Letters, vol. 18, pp. 165-177, 2010.

[6] J. L. Guardado, J. A. Flores, V. Venegas, J. L. Naredo, and F. A. Uribe, “A machine winding model for switching transient studies using network synthesis," IEEE Transactions on Energy Conversion, vol. 20, no. 2, pp. 322-328, 2005.

[7] M. K. Kazimierczuk, G. Sancineto, G. Grandi, U. Reggiani, and A. Massarini, "High-frequency smallsignal model of ferrite core inductors," IEEE Transactions on Magnetics, vol. 35, no. 5, pp. 4185-4191, 1999.

[8] S. Moreau, R. Kahoul, and J. P. Louis, "Parameters estimation of permanent magnetsynchronous machine without adding extra-signal input excitation," in Proceedings of the IEEE International Symposium on Industrial Electronics (IEEE-ISlE '04), pp. 371-376, May 2004.

[9] R. Kahoul, Y. Azzouz, and B. Ravelo, "Modelling of DC motors conducted low frequency EMI/EMC disturbance for automotive applications," European Journal of Scientific Research, vol. 63, no. 3, pp. 368386, 2011. 
[10] G. B. Huang, Q. Y. Zhu, and C. K. Siew, "Extreme learning machine: theory and applications," Neurocomputing, vol. 70, no. 1-3, pp. 489-501, 2006.

[11] Y. Xu, Z. Y. Dong, J. H. Zhao, P. Zhang, and K. P. Wong, "A reliable intelligent system for real-time dynamic security assessment of power systems," IEEE Transactions on Power Systems, vol. 27, no. 3, Article ID Article number6158623, pp. 1253-1263, 2012.

[12] S. Samet and A. Miri, "Privacy-preserving back-propagation and extreme learning machine algorithms," Data and Knowledge Engineering, vol. 79-80, pp. 40-61, 2012.

[13] Y. Song, J. Crowcroft, and J. Zhang, "Automatic epileptic seizure detection in EEGs based on optimized sample entropy and extreme learning machine," Journal of Neuroscience Methods, vol. 210, no. 2, pp. 132-146, 2012.

[14] S. Decherchi, P. Gastaldo, R. Zunino, E. Cambria, and J. Redi, “Circular-ELM for the reduced-reference assessment of perceived image quality," Neurocomputing, vol. 102, pp. 78-89, 2013.

[15] S. Decherchi, P. Gastaldo, A. Leoncini, and R. Zunino, "Efficient digital implementation of extreme learning machines for classification," IEEE Transactions on Circuits and Systems II, vol. 59, no. 8, Article ID Article number6236105, pp. 496-500, 2012.

[16] K. Choi, K.-A. Toh, and H. Byun, "Incremental face recognition for large-scale social network services," Pattern Recognition, vol. 45, no. 8, pp. 2868-2883, 2012.

[17] R. Minhas, A. A. Mohammed, and Q. M. Wu, "Incremental learning in human action recognition based on snippets," IEEE Transactions on Circuits and Systems for Video Technology, vol. PP, no. 99, 1 pages, 2011.

[18] G.-B. Huang, H. Zhou, X. Ding, and R. Zhang, "Extreme learning machine for regression and multiclass classification," IEEE Transactions on Systems, Man, and Cybernetics, Part B, vol. 42, no. 2, Article ID 6035797, pp. 513-529, 2012.

[19] F. Espinosa, J. A. Jiménez, E. Santiso et al., “Design and implementation of a portable electronic system for vehicle-driver-route activity measurement," Measurement, vol. 44, no. 2, pp. 326-337, 2011.

[20] Commission, I. E., CISPR 16-2-3 "Specification for radio disturbance and immunity measuring apparatus and methods Part 2-3: methods of measurement of disturbances and immunity—radiated disturbance measurements", 2008.

[21] C. Miyajima, Y. Nishiwaki, K. Ozawa, T. Wakita, K. Itou, and K. Takeda, "Cepstral analysis of driving behavioral signals for driver identification," in Proceedings of the IEEE International Conference on Acoustics, Speech and Signal Processing (ICASSP '06), pp. V921-V924, May 2006.

[22] W. Wang, W. Zhang, H. Guo, H. Bubb, and K. Ikeuchi, "A safety-based approaching behavioural model with various driving characteristics," Transportation Research Part C, vol. 19, no. 6, pp. 12021214, 2011.

[23] S. Alexandersson and M. Alaküla, "Automotive power electronic future-from an EMC perspective," in Proceedings of the International Symposium on Power Electronics, Electrical Drives, Automation and Motion (SPEEDAM '06), pp. 609-613, May 2006.

[24] L. De Santiago, F. Espinosa, M. A. Ruiz et al., "Effect of electrical vehicle-driver interaction on the radiated electromagnetic emissions: measurement methodology," in Proceedings of the IEEE-ICIT International Conference on Industrial Technology (ICIT '10), pp. 1113-1118, March 2010.

[25] C. Keller and K. Feser, "Fast emission measurement in time domain," IEEE Transactions on Electromagnetic Compatibility, vol. 49, no. 4, pp. 816-824, 2007.

[26] C. Keller and K. Feser, "A new method of emission measurement," in Proceedings of the IEEE International Symposium on Electromagnetic Compatibility (EMC '02), pp. 599-604, August 2002.

[27] C. Keller and K. Feser, "Fast emission measurement in time domain," in Proceedings of the 14th International Zurich Symposium On Electromagnetic Compatibility (EMC '01), Zurich, Switzerland, 2001.

[28] F. Krug, D. Mueller, and P. Russer, "Signal processing strategies with the TDEMI measurement system," IEEE Transactions on Instrumentation and Measurement, vol. 53, no. 5, pp. 1402-1408, 2004.

[29] G. B. Huang, D. H. Wang, and Y. Lan, "Extreme learning machines: a survey," International Journal of Machine Learning and Cybernetics, vol. 2, no. 2, pp. 107-122, 2011.

[30] G. B. Huang, L. Chen, and C. K. Siew, "Universal approximation using incremental constructive feedforward networks with random hidden nodes," IEEE Transactions on Neural Networks, vol. 17, no. 4, pp. 879-892, 2006.

[31] S. S. Haykin, Neural Networks: A Comprehensive foundation, Prentice Hall, 1999.

[32] M. T. Hagan, Neural Network Design, Brooks/Cole, 1996.

[33] C. M. Bishop, Neural Networks for Pattern Recognition, The Clarendon Press Oxford University Press, New York, NY, USA, 1995. 


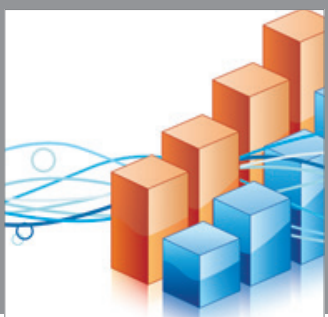

Advances in

Operations Research

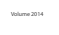

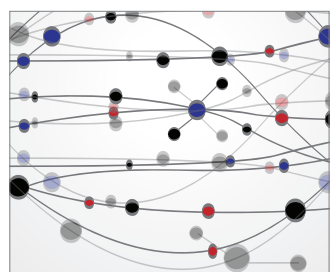

\section{The Scientific} World Journal
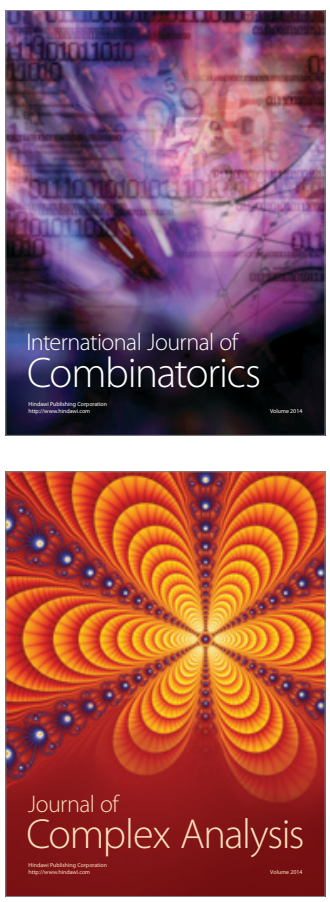

International Journal of

Mathematics and

Mathematical

Sciences
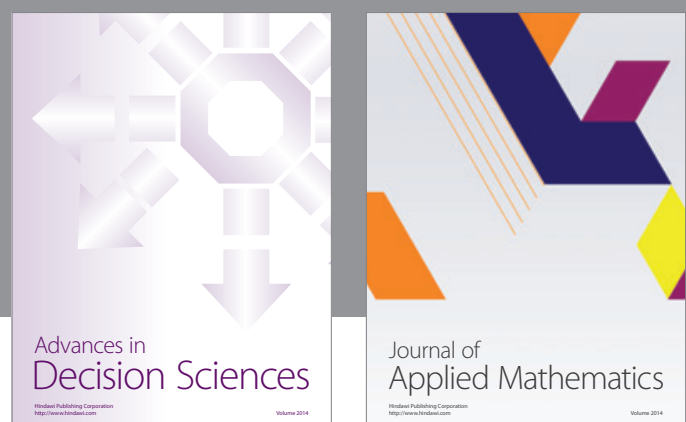

Journal of

Applied Mathematics
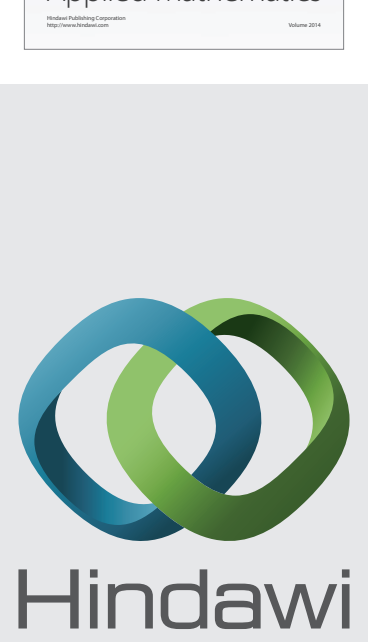

Submit your manuscripts at http://www.hindawi.com
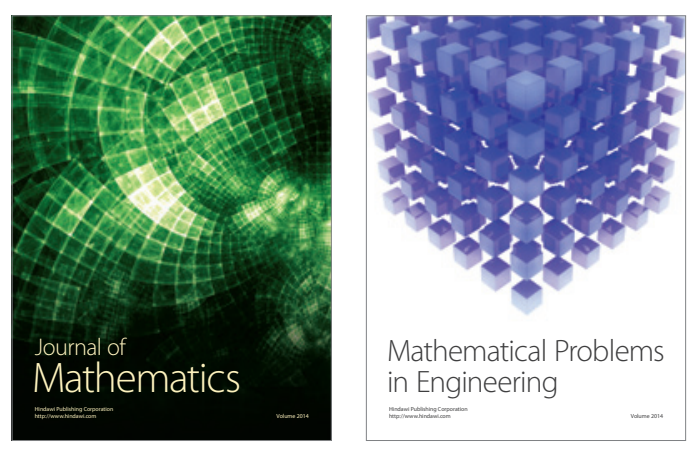

Mathematical Problems in Engineering
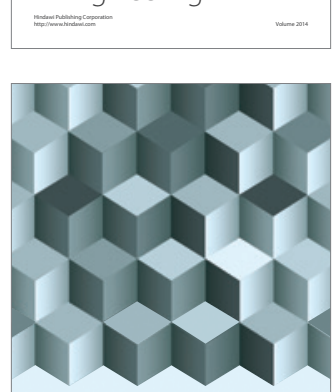

Journal of

Function Spaces
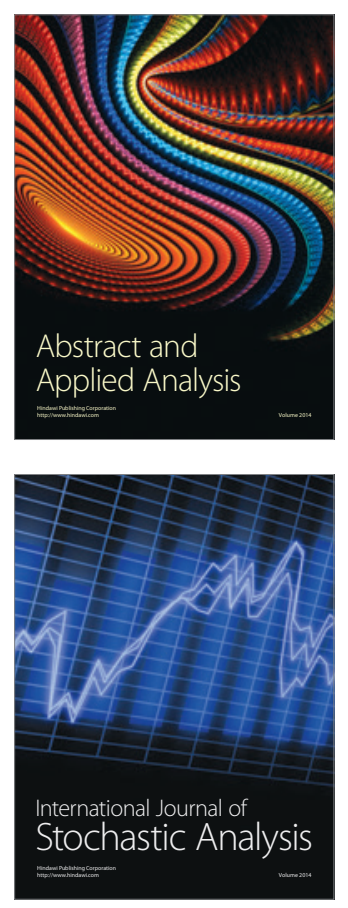

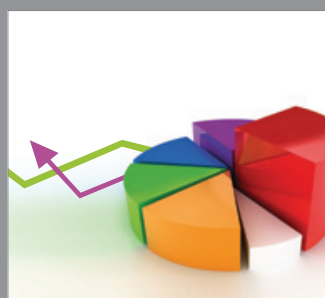

ournal of

Probability and Statistics

Promensencen
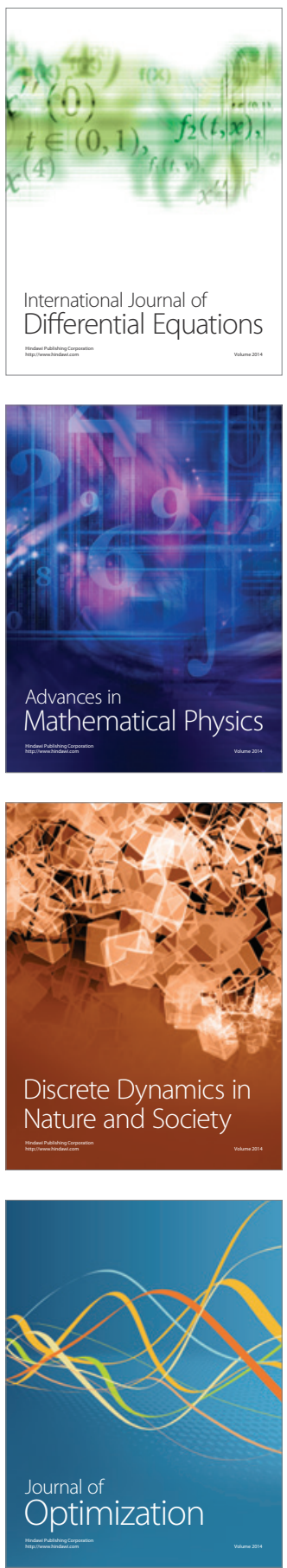